\title{
Bioactivity from Indonesian’s Marine Sponge Xestospongia Sp. as Antibacterial Resistance Escherichia coli
}

\section{Khairunnisa and Viqqi Kurnianda*}

Department Marine Science, Faculty Marine and Fisheries, Syiah Kuala University, Indonesia

\begin{abstract}
The isolation and characterization of bioactive compounds of Indonesian's marine sponge Xestospongia sp. has been done. Bioactive secondary metabolites alkaloid was isolated based on bioassay guided-separation with several steps of chromatography and monitored by the inhibition of antibacterial test of Escherichia coli. V15B02 compound is identified as the alkaloid compounds at $R_{f}$ value of 0.7 using a mobile phase of methanol: water 9:1 (v/v). FTIR spectrum data interpretation results showed that the compound of V15B02 has hydroxyl groups with their O-H stretching vibration in the area around $3425.58 \mathrm{~cm}^{-1}$ and $\mathrm{C}-\mathrm{N}$ vibration in the fingerprint region around $1111.00 \mathrm{~cm}^{-1}$. Their sharp band with sharp and strong intensity on the number of $2924.09 \mathrm{~cm}^{-1}$ is an aliphatic $\mathrm{C}-\mathrm{H}$. That this was reinforced by a sharp uptake and up down in numbers of $1404.18 \mathrm{~cm}^{-1}$ which is $\mathrm{C}-\mathrm{H}$ band indicates the terminal methyl groups. In the catchment area $1636.64 \mathrm{~cm}^{-1}$ showed the presence of $\mathrm{C}=\mathrm{O}$ group. Indication of a cyclic amine group of alkaloids visible from the drop of vibration tertiary $\mathrm{N}$ in the area around $1165.00 \mathrm{~cm}^{-1}$. V15B02 compounds have an activity inhibiting the growth of bacterial resistance Escherichia coli at a concentration of $70 \mathrm{ug} / \mathrm{mL}$.
\end{abstract}

Keywords: Alkaloid; Indonesian's marine sponge; Escherichia coli resistance

\section{Introduction}

Disease caused by the bacterial Escherichia coli is very dangerous to the health of all human beings. It has been reported that every year as many as 2 million people are victims of this disease [1].

At the present time, many studies have focused on finding compounds that have the potential of natural ingredients to the bacterial Escherichia coli, but the results obtained are not maximized because the bacteria are able to evolve so resistance to antibiotics [2-4].

One potential source of compounds is sponge. The resulting sponge bioactivity are compounds that are toxic. Sponge metabolite active against some cells and microorganisms are pathogens that can be used as a basic ingredient in the search for new drugs that are very useful for the development of research, medical and medicine $[5,6]$.

Therefore, in this study will be described on the isolation and characteristics of alkaloid compounds result of secondary metabolites of sponge A15F04 and activity of isolated compounds as antibacterial compounds.

\section{Experimental}

HPLC data was obtained on a HPLC Shimadzu C196-E061R Prominence using a C18 column with a detector Photodiode Array (PDA). IR spectrum was recorded on a FTIR Shimadzu IR Prestige 21.

\section{Chemical and reagents}

Methanol, ethyl acetate, chloroform, dichloromethane, $n$-hexane, Dragendorff reagents, cerium sulfate reagents, nutrient agar medium (NA), bacterial resistant Escherichia coli, chloramphenicol and $\mathrm{H}_{2} \mathrm{O}$.

\section{Specimen of sponge}

Sponge taken at Aramanyang, Indonesia in 2015. Deposit of sponge retrieved and stored in Laboratorium of Marine Chemistry, Department of Marine Science, Faculty of Marine and Fisheries. Sponges analyzed in UPT LTSIT, Lampung University (Indonesia).

\section{Specimen of mycobacterial}

Mycobacterial resistance Escherichia coli taken from RSUD Zainoel
Abidin's patient (Aceh, Indonesia) and cultured in Mycrobacterium and Pharmaceutical's Laboratory (Aceh). Then the strain of mycobacterial resistance Escherichia coli was grown in Nutrient Agar medium (NA) at temperature $37^{\circ} \mathrm{C}$ [7].

\section{Extraction and isolation}

The sponge (600 g, wet weight) was cut into small pieces, and extracted with $\mathrm{MeOH}(3 \mathrm{~L} \times 3)$. Crude samples of sponge $(32 \mathrm{~g})$ partitioned twice. First, extract coarse sponge partitioned in a mixture of methanol and $n$-hexane. The solution was shaken several times in a separating funnel, and then allowed to stand to form two fractions. Each fraction was separated and repetition partition with dichloromethane:methanol. Third fraction separation results obtained results concentrated by rotary evaporator until dry and then determined the fraction obtained quantitative weight of each extract. Sponge extracts second partition that is using the reagent ethyl acetate and methanol.

Then TLC analysis is performed by means of a silica bit samples on TLC plates and eluted with a mixed solvent as the eluent. Elution is carried out in a sealed container so that the eluent is saturated. Then, the resulting chromatogram, observed with a UV lamp. Alkaloid content analysis components in the sample used TLC visualization reagents specific test. TLC visualization test reagent used is a reagent Dragendorff and characterized by the onset of orange-red stain on a TLC plate. As for knowing the organic compound is generally used in a sample of cerium sulfate reagent yag marked with blackish brown stain [8].

Sponge extracts fractionated receipts column chromatography used as the stationary phase and elution was carried out correctly by comparison a suitable solvent system. The existence of the components

*Corresponding author: Viqqi Kurnianda, Department Marine Science, Faculty Marine and Fisheries, Syiah Kuala University, Indonesia, Tel: +62-821-7874-2727 E-mail:60khairunnisa@gmail.com, viqqikurnianda@yahoo.co.id

Received April 19, 2017; Accepted April 25, 2017; Published May 03, 2017

Citation: Khairunnisa, Kurnianda V (2017) Bioactivity from Indonesian's Marine Sponge Xestospongia Sp. as Antibacterial Resistance Escherichia coli. Nat Prod Chem Res 5: 265. doi: 10.4172/2459-6836.1000265

Copyright: (c 2017 Khairunnisa, et al. This is an open-access article distributed under the terms of the Creative Commons Attribution License, which permits unrestricted use, distribution, and reproduction in any medium, provided the original author and source are credited. 
of the fractions obtained re monitored by TLC method. The fraction that has the same $R_{f}$ value will be combined into a single faction. Positive fraction of alkaloids and have the greatest abundance would be defined as an advanced analysis of samples, and then purified.

\section{Detection methods}

The HPLC analysis using a C18 column, detector Photo Diodes Aray (PDA) eluted with methanol: water (9:1). The results of these HPLC chromatograms be further analyzed based on the peaks are formed. Infrared resonance analysis characterization of functional groups in the sample used an FTIR spectrophotometer.

\section{Test of bioassay}

Test of Antibacterial method using diffusion agar with Nutrient Agar medium (NA) was added to mycobacterial resistance Escherichia coli then added $2 \%$ DMSO as a negative control. The active compound and chloramphenicol as a comparison.

\section{Result and Discussion}

Preliminary Analysis of Thin Layer Chromatography at Xestospongia sp. as A15F04. Alkaloid compound that would be isolated from A15F04 sponge extracts were analyzed by a thin layer of chromatography (TLC). TLC test aims to determine the existence of the components of the compound in the sample. The existence of the alkaloid compounds are identified using Dragendorff reagents by using C18 stationary phase and a mobile phase of methanol which is characterized by patches of orange on a TLC plate. The results of TLC analysis showed that the methanol extract of the sponge contains component of polar alkaloid and non-polar alkaloid. Polar alkaloid compound showed spot at $\mathrm{R}_{f}$ value of 0.7 , while the non-polar alkaloid compound indicated spots at $\mathrm{Rf}$ value 0 .

\section{Separation alkaloid compounds using column chromatography}

Methanol fraction from column chromatography using preparative C18 stationary phase and a mobile phase of methanol: water (4:1) yielded 3 fractions. The first fraction as V03B25 is dark yellow colour , the second fraction as V03B26 is brown colour and third fraction as V03B27 is yellow colour. Further, the TLC test results of the three fractions in a row shows that all positive fractions alkaloid with orange stains on Dragendorff visualization reagent (Table 1).

Furthermore, the first and second fractions concentrated by rotary evaporator obtained the dry weight ratio of the first fraction : the fraction of a second with a ratio of 1:6. The second fraction extracted with dichloromethane : methanol (1:1) v/v so that the faction would be dissolved in part. Residual fraction was then dissolved in water. Extraction with dichloromethane and methanol compounds aimed at separating the compound from the salt content. Salt contained in the compounds can affect the properties of these compounds for bioactivity of salt can make as if the compound has antibacterial activity [9].

Extract from dichloromethane: methanol and water at each of the fractions are analyzed by TLC silica gel using UV lamps and Dragendorff visualization. The first fraction from dichloromethane: methanol extract as V03B25 is alkaloid compounds identified in the value of $R_{f} 0.8$. The level of purity between fractions V03B25 and V03B26 analyzed by HPLC using a C18 column, detector Photo Diodes Aray (PDA) eluted with methanol : water (9:1) v/v. Results of the analysis showed a peak fraction V03B25 little wide on the retention time of 1.3 minutes and a high peak at 1.8 minutes. While on V03B26 fraction showed two absorption peaks at retention time of 1.8 minutes and 2.3 minutes. The formation of two absorption peaks on each of the fractions indicating that the compound is not pure and necessary further purification (Figure 1).

Based on HPLC analysis that the fraction V03B26 has two absorption peaks. Therefore, the fraction was purified by column chromatography. OPN C18 stationary phase used and the mobile phase of water and methanol: water $(3: 1)$. Fraction of water has resulted in 13 fractions and fractions of methanol: water (3:1) resulted in 9 fractions were then simplified into six fractions based on those observations TLC test alkaloid compounds in each fraction. Furthermore, both of fractions are analyzed using the TLC plate $\mathrm{C} 18$ and the eluent methanol: water (9:1). V07B32 water fraction alkaloid compounds identified in the value of $\mathrm{R}_{\mathrm{f}} 0.7$ and V07B33 a fraction of methanol: water (3:1) as alkaloids identified.

Fraction V07B32 re-extracted with methanol and purified by column chromatography using OPN C18 and water eluent. Purified fraction from V07B32 obtained weight $5 \mathrm{mg}$ as V15B02. TLC test showed the presence of the alkaloid compounds spots at $R_{f}$ value of 0.7 using a plate $\mathrm{C} 18$ and the eluent methanol: water (9:1). The observation with ultraviolet light shows the fraction V15B02 a UV active compounds. Compounds were tested for bioactivity V15B02 antibacterial and characterized using infrared spectrophotometry.

\section{FTIR analysis}

Infrared spectrophotometry is used to characterize the functional groups contained in the compound V15B02. The results of the analysis of the FTIR spectrum can be seen in the Figure 2. Characterization of functional groups contained in the compound V15B02 showed that this compound has a hydroxy group in the presence of $\mathrm{O}-\mathrm{H}$ stretching vibration is widened in the area around $3425.58 \mathrm{~cm}^{-1}$ and the vibration $\mathrm{C}-\mathrm{N}$ in the area around $1111.00 \mathrm{~cm}^{-1}$, Their sharp band with sharp and strong intensity on the number $2924.09 \mathrm{~cm}^{-1}$ is an aliphatic $\mathrm{C}-\mathrm{H}$, this was reinforced by a sharp uptake and weak in numbers $1404.18 \mathrm{~cm}^{-1}$ which is $\mathrm{C}-\mathrm{H}$ bend indicates the terminal methyl groups. In the catchment area $1636.64 \mathrm{~cm}^{-1}$ showed the presence of $\mathrm{C}=\mathrm{O}$ groups. Further analysis of the fingerprint region showed the presence of alkaloids which saw

\begin{tabular}{|c|c|c|}
\hline Sample & Concentrasi $(\mathbf{u g} / \mathrm{mL})$ & Inhibition Zone \\
\hline Metanol : water & - & - \\
\hline V15B02 & 70 & + \\
\hline DMSO 2\% & 30 & +++ \\
\hline
\end{tabular}

Table 1: Inhibition from fraction V15B02.

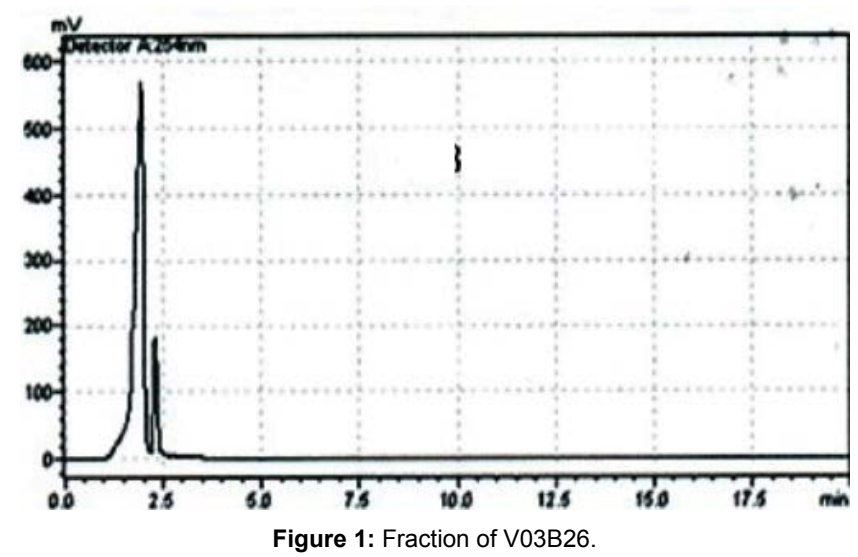


Citation: Khairunnisa, Kurnianda V (2017) Bioactivity from Indonesian's Marine Sponge Xestospongia Sp. as Antibacterial Resistance Escherichia coli. Nat Prod Chem Res 5: 265. doi: 10.4172/2459-6836.1000265
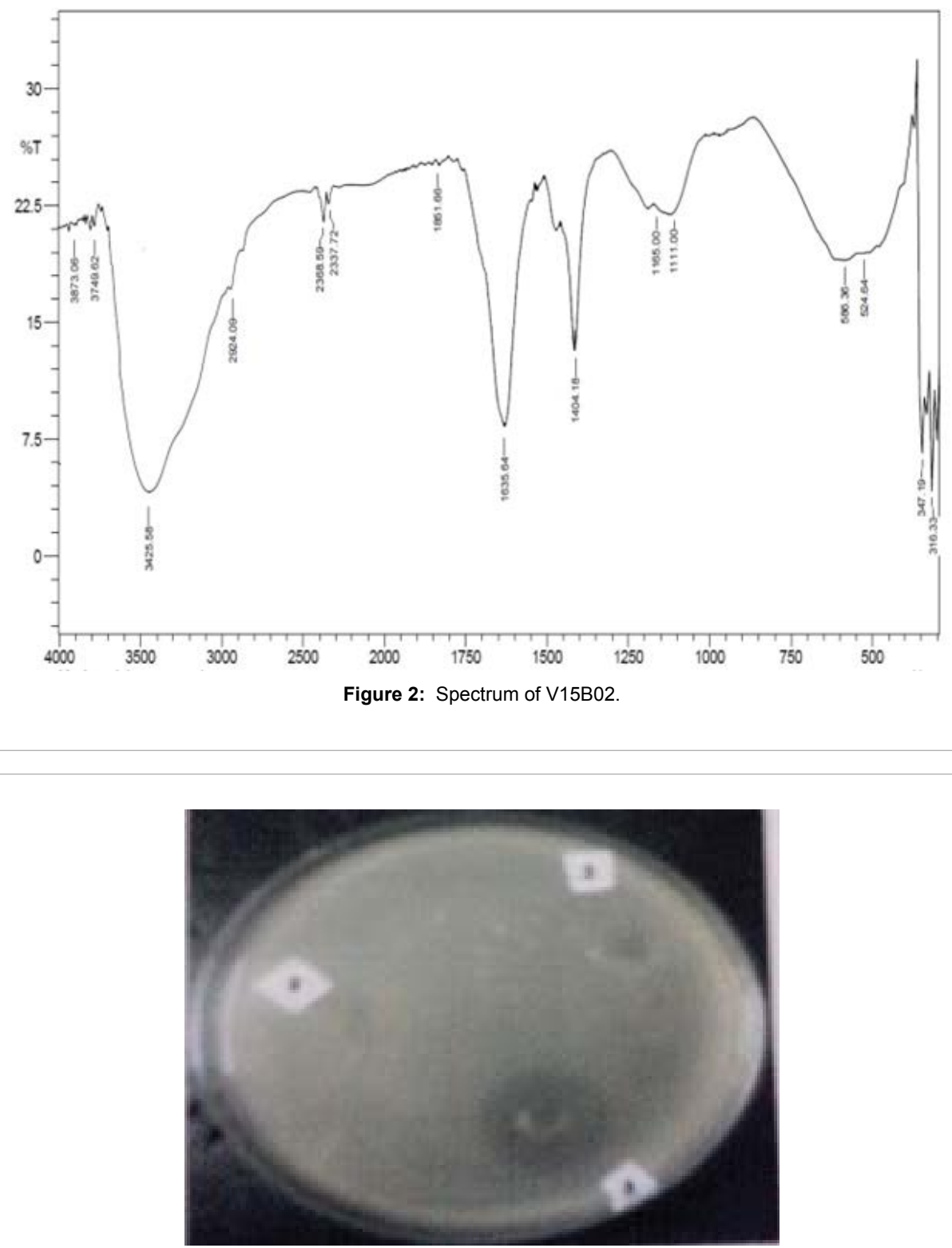

Figure 3: Bioactivity from fraction V15B02 with (1) Methanol: water, (2) V15B02 and (3) DMSO 2\%.

their vibration tertiary $\mathrm{N}$ on the area around $1165.00 \mathrm{~cm}^{-1}$ which indicate a cyclic amine group of alkaloids $[9,10]$.

\section{Bioassay test}

Bioassay tested of antibacterial from V15B02 fraction against the inhibition of the growth of bacterial resistant Escherichia coli. Methanol: water $(7: 3)$ used as a standard test negative for antibacterial activity and DMSO 2\% used as a positive control (Figure 3).

The test results show that the bioactivity from V15B02 compounds have activity in inhibiting the growth of bacterial resistant Escherichia coli. It is observed from the formation of a clear zone on the test medium. The antibacterial activity possessed V15B02 compounds can be caused by clusters contained in the compound, for example a tertiary amine group and a hydroxyl group [9].

\section{Conclusions}

This study has founded compound for inhibiting the growth of bacterial resistant Escherichia coli with concentration $70 \mathrm{ug} / \mathrm{mL}$.

\section{Acknowledgements}

LIPI for FTIR and UPT Laboratorium Terpadu dan Sentra Inovasi Teknologi (Indonesia) for bioassay experiment.

\section{References}

1. World Health Organization (2014) Global Health Observatory. August 27, 2014

2. Gotsbacher MP, Karuso P (2015) New Antimicrobial Bromotyrosine Analogues from the Sponge Pseudoceratina purpurea and Its Predator Tylodina corticalis. Marine Drugs 13: 1389-1409.

3. Diaa TA, Lamiaa AS, Hani ZA (2013) Bioactive Compounds from the Red Sea Marine Sponge Hyrtios Species. Marine Drugs 11: 1061-1070. 
Citation: Khairunnisa, Kurnianda V (2017) Bioactivity from Indonesian's Marine Sponge Xestospongia Sp. as Antibacterial Resistance Escherichia coli. Nat Prod Chem Res 5: 265. doi: 10.4172/2459-6836.1000265

4. Valentin B, Vinod V, Beulah CM (2011) Biopotential of secondary metabolites isolated from marine sponge Dendrilla nigra. Asian Pacif J Trop Dis 299-303.

5. Kobayashi M, Higuchi K, Murakami N, Tajima H, Aoki S (1997) Callystatin A, a potent cytotoxic polyketide from the marine sponge. Callyspongia Tett Lett 38: 2859-2862.

6. Berer N, Rudi A, Goldberg I, Benayahu Y, Kashman Y (2004) Callynormine A, a new marine cyclic peptide of a novel class. J Org Lett 6: 2543-2545.

7. Mbah JA, Ngemenya MN, Abawah AL, Babiaka SB, Nubed LN, et al. (2012)
Bioassay-guided discovery of antibacterial agents: in vitro screening of Peperomia vulcanica, Peperomia fernandopoioana and Scleria striatinux. Ann Clin Microbiol Antimicrob 11:10.

8. Harborne JB (1984) Phytochemical Method. Chapman and Hall Itd. London.

9. Huang RM, MaW, Jun-De D, Xue-Feng Z, Xu T, et al. (2010). A New 1,4-Diazepine from South China Sea Marine Sponge Callyspongia Species. Molecules 15: 871-877.

10. McMurry J (2008) Organic Chemistry. $7^{\text {th }}$ edn. Graphic World Inc. pp: 440-469. 